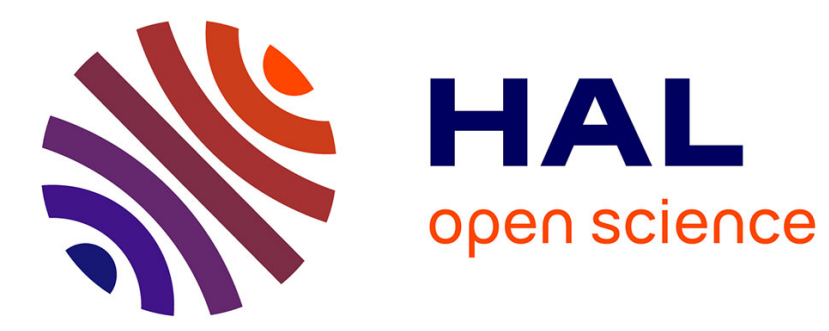

\title{
Unknown input observer for LPV systems
}

Benoît Marx, Dalil Ichalal, José Ragot, Didier Maquin, Saïd Mammar

\section{To cite this version:}

Benoît Marx, Dalil Ichalal, José Ragot, Didier Maquin, Saïd Mammar. Unknown input observer for LPV systems. Automatica, 2019, 100, pp.67-74. 10.1016/j.automatica.2018.10.054 . hal-01931453

\section{HAL Id: hal-01931453 \\ https://hal.univ-lorraine.fr/hal-01931453}

Submitted on 17 Apr 2019

HAL is a multi-disciplinary open access archive for the deposit and dissemination of scientific research documents, whether they are published or not. The documents may come from teaching and research institutions in France or abroad, or from public or private research centers.
L'archive ouverte pluridisciplinaire HAL, est destinée au dépôt et à la diffusion de documents scientifiques de niveau recherche, publiés ou non, émanant des établissements d'enseignement et de recherche français ou étrangers, des laboratoires publics ou privés. 


\title{
Unknown input observer for LPV systems
}

\author{
Benoît Marx $^{\mathrm{a}, *}$, Dalil Ichalal ${ }^{\mathrm{b}}$, José Ragot $^{\mathrm{a}}$, Didier Maquin $^{\mathrm{a}}$, Saïd Mammar ${ }^{\mathrm{b}}$ \\ ${ }^{a}$ Université de Lorraine, CRAN, UMR 7039, 2 avenue de la Foret de Haye, 54516 Vandoeuvre-les-Nancy, France and CNRS, CRAN, UMR 7039, France \\ ${ }^{b}$ IBISC-Lab, Évry Val d'Essonne University, 40, rue de Pelvoux, 91020, Courcouronnes Cedex, France
}

\begin{abstract}
This paper proposes two improvements in the design of unknown input observers (UIO) for linear parameter varying (LPV) systems. First, the parameter dependency of the UIO is not restricted to mimic the one of the system in order to relax the existing decoupling conditions. Second, the output equation of the LPV systems is not supposed to be linear time invariant. Both perfect UI decoupling or mixed decoupling and attenuation are considered, whether the relaxed structural conditions are met or not.
\end{abstract}

Keywords: Unknown input observer, LPV systems, fault estimation.

\section{Introduction}

Since in practical situations, all process inputs are rarely known, unknown input observers (UIO) are essential in process control and diagnosis and have received a great attention. An UIO provides the system state estimate, even in the presence of unknown inputs (UI) encompassing faults, perturbations, modeling uncertainties, etc. The several proposed approaches can be split into two classes. In the first one, the state estimation is decoupled from the UI thanks to structural conditions (Darouach et al., 1994) or by some state transformations (Hou and Muller, 1994; Aldeen and Sharma, 2008; Tan et al., 2008). In the second one, the system state is usually augmented with the UI and, under some assumptions on the time variations of the UI, an augmented observer may provide simultaneous state and UI estimation. The so-called PI observer, proposed by Wojciechowski (1978) for linear systems affected by constant UI, has been extended to descriptor and nonlinear systems affected by time polynomial UI (Koenig, 2006; Gao and Ho, 2004; Ichalal et al., 2009). The original linear UIO proposed by Darouach et al. (1994) has been extended to nonlinear polytopic systems by duplicating the polytopic structure of the system in the nonlinear UIO. Therefore, the structure of the UIO is fixed a priori and asymptotic convergence of the state estimation error towards zero is ensured by solving Lyapunov based LMI conditions (Marx et al., 2007; Chadli and Karimi, 2013). Despite the appealing simplicity of this approach, the duplication of the polytopic structure of the system in the UIO drastically reduces the system class for which such an observer can be designed (Ichalal and Mammar, 2015; Ichalal et al., 2015). For example, the UIO design may fail even if the system is strongly algebraically observable or at least strongly detectable.

\footnotetext{
${ }^{*}$ Corresponding author

Email addresses: benoit.marx@univ-lorraine.fr (Benoît Marx), dalil.ichalal@ibisc.univ-evry.fr (Dalil Ichalal), jose.ragot@univ-lorraine.fr (José Ragot), didier .maquin@univ-lorraine.fr (Didier Maquin), said.mammar@ibisc.univ-evry.fr (Saïd Mammar)
}

In this paper a new LPV UIO is presented. Its polytopic structure does not necessarily mimic the system structure. It allows to design UIO for a larger class of systems by avoiding restrictive decoupling conditions (as those of Marx et al. (2007); Chadli and Karimi (2013); Hassanabadi et al. (2016)). This result is obtained by postponing the use of the polytopic transformation of a general LPV form into a polytopic one. It results in more degrees of freedom in the UIO design by not searching for a common solution to several equality constraints imposed by the decoupling between state the estimation and the UI (Chadli and Karimi, 2013). Moreover, all the published works on UIO for polytopic or LPV systems consider linear time invariant output equations and cannot be applied to systems with LPV outputs (Marx et al., 2007; Chadli and Karimi, 2013; Hassanabadi et al., 2016; Heemels et al., 2010). The proposed UIO structure allows to overcome this limitation and provides a solution for systems with LPV output equations. In the presence of disturbances, the UIO is designed to ensure perfect fault decoupling and asymptotic convergence in an origin centered ball which radius is minimized. If no disturbances affect the system, then the state estimation error asymptotically converges to zero and the fault estimation is also provided.

The paper is organized as follows. The problem statement and some useful materials are provided in the Section 2. The UIO design for LPV systems affected by fault UI and disturbance UI is detailed in Section 3. Before concluding, some illustrative examples are provided in Section 4.

\section{Problem statement and preliminaries}

\subsection{Notations}

In the remainder of the paper, the following notations are used. For any square matrix, $X>0$ (resp. $X<0$ ) means that $X$ is positive (resp. negative) definite and $\mathbb{S}$ stands for $\mathbb{S}(X)=X+X^{T}$. The minimal and maximal eigenvalues of $X$ are respectively denoted $\underline{\lambda}(X)$ and $\bar{\lambda}(X)$. For any matrix $X$ (not 
necessarily square), $X^{T}$ and $X^{+}$respectively denote the transpose and the left pseudo inverse of $X . I_{n} \in \mathbb{R}^{n \times n}$ is the identity matrix. The Euclidean norm of a vector $x(t)$ is denoted $\|x(t)\|_{2}$, and $\|x(t)\|_{\infty}$ denotes the supremum norm defined by $\|x(t)\|_{\infty}=\sup _{t \geq 0}\{|x(t)|\}$. A parameter dependent matrix $X(\rho(t))$ is shortened in $X(\rho)$ and its first time derivative, depending on $\rho(t)$ and $\dot{\rho}(t)$, is denoted $\dot{X}(\rho, \dot{\rho})$. A set of functions $\mu_{i}$ (for $i=1, \ldots, r)$ is said to satisfy the convex sum property, if the constraints (1) hold for $i=1, \ldots, r$ and for all $t \geq 0$.

$$
0 \leq \mu_{i}(\xi(t)) \leq 1 \quad \sum_{i=1}^{r} \mu_{i}(\xi(t))=1
$$

Simple and double polytopic sums of matrices $X_{i}$ and $X_{i j}$, with functions $\mu_{i}$ satisfying (1) are denoted as follows

$$
X_{\mu}=\sum_{i=1}^{r} \mu_{i}(\xi(t)) X_{i} \quad X_{\mu \mu}=\sum_{i=1}^{r} \sum_{j=1}^{r} \mu_{i}(\xi(t)) \mu_{j}(\xi(t)) X_{i j}
$$

For given positive integers $p$ and $r$ ( $r$ being the number of functions $\left.\mu_{i}\right)$, the integer set $\mathcal{I}_{r}$ is defined by $\mathcal{I}_{r}=\{1,2, \ldots, r\}$ and the vector sets $\mathbb{I}_{p, r}$ and $\mathbb{I}_{p, r}^{+}$are defined by

$$
\begin{aligned}
& \mathbb{I}_{p, r}=\left\{i=\left(i_{1} i_{2} \ldots i_{p}\right)^{T}, i_{j} \in \mathcal{I}_{r}, j \in \mathcal{I}_{p}\right\} \\
& \mathbb{I}_{p, r}^{+}=\left\{i \in \mathbb{I}_{p, r}, i_{k} \leq i_{k+1}, k \in \mathcal{I}_{p-1}\right\}
\end{aligned}
$$

For $i \in \mathbb{I}_{p, r}$, the vector subset $\mathcal{P}(i) \subset \mathbb{I}_{p, r}$ denotes the vector set composed of $i$ and its possible permutations Sala and Ariño (2007).

\subsection{Problem statement}

Consider the following LPV system with unknown inputs

$$
\begin{aligned}
& \dot{x}(t)=A(\rho) x(t)+B(\rho) u(t)+F(\rho) f(t)+E(\rho) w(t) \\
& y(t)=C(\rho) x(t)
\end{aligned}
$$

where $x(t) \in \mathcal{X} \subset \mathbb{R}^{n}, u(t) \in \mathcal{U} \subset \mathbb{R}^{n_{u}}, f(t) \in \mathcal{F} \subset \mathbb{R}^{n_{f}}, w(t) \in$ $\mathcal{W} \subset \mathbb{R}^{n_{w}}$ and $y(t) \in \mathcal{Y} \subset \mathbb{R}^{n_{y}}$ respectively denote the state variables, the known control inputs, the fault unknown inputs, the disturbance unknown inputs and the measured outputs. The system matrices depend on the time varying parameter $\rho(t) \epsilon$ $\mathcal{R} \subset \mathbb{R}^{n_{\rho}}$. The following assumptions are made.

Assumption 1. For all $t \geq 0$, the time varying parameter $\rho(t)$ and its derivative $\dot{\rho}(t)$ are bounded and real time accessible. The matrices $A(\rho), B(\rho), C(\rho), E(\rho)$ and $F(\rho)$ are bounded for all possible values of $\rho$.

Assumption 2. For all $\rho \in \mathcal{R}$, the matrices $C(\rho)$ and $F(\rho)$ in (4) satisfy the rank condition: $\operatorname{rank}(C(\rho) F(\rho))=\operatorname{rank}(F(\rho))$.

Assumption 3. For all $t \geq 0, w(t)$ is such that $\|w(t)\|_{2}<\bar{w}$, where $\bar{w}$ is known.

Assumption 4. The time derivative matrices $\dot{H}(\rho, \dot{\rho})$ and $\dot{C}(\rho, \dot{\rho})$ are bounded for all possible values of $\rho$ and $\dot{\rho}$, with $H(\rho)=-F(\rho)(C(\rho) F(\rho))^{+}$.
Remark 1. The assumption of a real time accessible parameter $\rho(t)$ is classical in the LPV or polytopic frameworks (Sename et al., 2013; Guerra et al., 2015; Lendek et al., 2010; Tanaka and Wang, 2001; Tuan et al., 2001). Assuming that its first time derivative is available is also quite realistic since it may be provided by direct measurements (e.g. angular position and velocity sensors, or speed and acceleration sensors (Yacine et al., 2015)). If such sensors are not available, high order sliding mode differentiators (Levant, 2003) or algebraic differentiators (Fliess et al., 2008) can be used to estimate the parameter derivative in finite time. The known convergence time can then be used as an initial time for the parameter derivative and for the UIO.

The proposed UIO is defined by

$$
\begin{aligned}
& \dot{z}(t)=N(\rho, \dot{\rho}) z(t)+G(\rho) u(t)+L(\rho, \dot{\rho}) y(t) \\
& \hat{x}(t)=z(t)-H(\rho) y(t)
\end{aligned}
$$

where the parameter dependent matrices $N(\rho, \dot{\rho}), G(\rho), L(\rho, \dot{\rho})$ and $H(\rho)$ are computed in order that the state estimation error between (4) and (5), denoted $e(t)$ and defined by $e(t)=\hat{x}(t)-x(t)$ converges towards zero when $w(t)$ is identically null, or at least in a bounded ball in the presence of $w(t)$. These results are established with the help of the Lyapunov theory and the inputto-state stability (ISS) (Sontag and Wang, 1995). The UIO existence conditions are expressed as LMI conditions.

\subsection{Useful material}

Most works on UIO design for LPV systems consider a polytopic representation of the LPV system.

Lemma 1. Tanaka and Wang (2001); Lendek et al. (2010) Under Assumption 1, a parameter dependent matrix can be written as a polytopic matrix, thanks to the polytopic transformation or sector nonlinearity, and the LPV system (4) can thus be exactly rewritten as a polytopic system (6), at least on a compact set of $\mathcal{X} \times \mathcal{U} \times \mathcal{F} \times \mathcal{W}$

$$
\begin{aligned}
& \dot{x}(t)=A_{\mu} x(t)+B_{\mu} u(t)+F_{\mu} f(t)+E_{\mu} w(t) \\
& y(t)=C_{\mu} x(t)
\end{aligned}
$$

where the matrices $A_{i}, B_{i}, F_{i}, E_{i}$ and $C_{i}$ (for $i \in \mathcal{I}_{r}$ ) used in (2), are constant. The functions $\mu_{i}$ depend on the premise variable $\xi(t)$ which depends on the parameter $\rho(t)$ of the LPV system (4).

Once the system and the observer are written in polytopic form, the UIO design reduces to the stability analysis of the estimation error. With the help of Lyapunov theory, it reduces to check the negativity of a polytopic matrix sum: $X_{\mu \mu}<0$. To do so, many studies proposed to relax the intuitive but restrictive requirement $X_{i j}<0$. Some overviews are available in Feng (2006); Sala (2009); Lendek et al. (2010). In Ichalal and Mammar (2015) we used the popular Tuan's relaxation scheme Tuan et al. (2001), which can be considered as a special case of the more general approach proposed by Sala and Ariño (2007). This latter approach will be used in the present paper and is recalled in Lemma 2. 
Lemma 2. Sala and Ariño (2007) For a given integer satisfying $p \geq 2$, the inequality $X_{\mu \mu}<0$ is implied by

$$
\sum_{j \in \mathcal{P}(i)} X_{j_{1} j_{2}}<0, \forall i \in \mathbb{I}_{p, r}^{+}
$$

where $j_{1}$ and $j_{2}$ are the first and second components of $j \in \mathbb{R}^{p}$.

\subsection{Background on UIO for LPV systems}

In the existing works on UIO for LPV systems with $w(t)=0$ (Chadli and Karimi, 2013; Marx et al., 2007), first (4) is written as a polytopic system (6). Since the considered systems have LTI output equation, $C(\rho(t))=C$ holds in (4) and $C_{i}=C$ in (6b). Consequently, the UIO is usually defined as a polytopic system with the matrix $H$ being LTI as the matrix $C$ of the system. Thus, the UIO reduces to

$$
\begin{aligned}
& \dot{z}(t)=N_{\mu} z(t)+G_{\mu} u(t)+L_{\mu} y(t) \\
& \hat{x}(t)=z(t)-H y(t)
\end{aligned}
$$

In order to make the state estimation error independent of the UI, the decoupling condition imposes to find a matrix $H$ that satisfies: $H C F_{\mu}=-F_{\mu}$. This equality constraint can be equivalently written as the following rank condition

$$
\operatorname{rank}\left(C F_{\mu}\right)=\operatorname{rank}\left(F_{\mu}\right)
$$

Taking into account the positivity of the weighting functions $\mu_{i}$, a sufficient existence condition of such a matrix $H$ is

$$
\operatorname{rank}\left(C\left[\begin{array}{lllll}
F_{1} & F_{2} & \ldots & F_{r}
\end{array}\right]\right)=\operatorname{rank}\left(\left[\begin{array}{lllll}
F_{1} & F_{2} & \ldots & F_{r}
\end{array}\right]\right)
$$

This rank condition, limited to the special case of LTI output, corresponds to the one in Chadli and Karimi (2013); Marx et al. (2007). In Ichalal and Mammar (2015); Ichalal et al. (2015), it is pointed that if the system (6) and the observer (8) share the same polytopic structure, then the decoupling condition to obtain an unique matrix $H$ satisfying the $r$ constraints $H C F_{i}=$ $-F_{i}$, for $i \in \mathcal{I}_{r}$, may become restrictive and then limits the applicability of the UIO design as it will be illustrated in the Example 1. Moreover this UIO structure cannot be generalized for LPV systems with LPV output equation.

\section{Unknown Input Observer design}

In this section, the design of the UIO (5) for (4) is detailed. The first objective of the UIO design is to perfectly decouple the state estimation from the faults $f$. Secondly, the influence of the disturbance $w$ is minimized by ensuring the state estimation error to asymptotically converge in an origin-centered ball, using the ISS. The ball radius is minimized when computing the observer gains by LMI optimization. Thirdly, the proposed UIO design brings some relaxation thanks to the use of a parameter dependent matrix $H(\rho)$ instead of a constant $H$. Since the use of the polytopic transformation is postponed, it results in less restrictive decoupling conditions needed to ensure
$H(\rho) C(\rho) F(\rho)=-F(\rho)$. Indeed, when seeking for rank condition after having used the polytopic transformation, an important part of the information embedded in the weighting functions $\mu_{i}$ is ignored, since only their positivity is used. Consequently sufficient and restrictive conditions are obtained. Here, the parameter dependent form of $F(\rho)$ and $C(\rho)$ is kept and $H(\rho)$ is analytically deduced. The UIO design procedure is given in the following theorem.

Theorem 1. Provided that the system (4) satisfies the Assumptions 1, 2, 3 and 4, define the parameter dependent matrices $H(\rho), P(\rho)$ and $\mathcal{A}(\rho, \dot{\rho})$ by

$$
\begin{aligned}
H(\rho) & =-F(\rho)(C(\rho) F(\rho))^{+} \\
P(\rho) & =I_{n}+H(\rho) C(\rho) \\
\mathcal{A}(\rho, \dot{\rho}) & =\dot{P}(\rho, \dot{\rho})+P(\rho) A(\rho)
\end{aligned}
$$

and apply the polytopic transformation to $C(\rho), \mathcal{A}(\rho, \dot{\rho})$ and $-P(\rho) E(\rho)$ to obtain: $C(\rho)=C_{\mu}, \mathcal{A}(\rho, \dot{\rho})=\mathcal{A}_{\mu}$ and $-P(\rho) E(\rho)=\mathcal{E}_{\mu}$. For given real positive scalars $\alpha$ and $\alpha_{1}$ and for a given integer $p \geq 2$, if there exists a symmetric positive definite matrix $X \in \mathbb{R}^{n \times n}$, matrices $\bar{K}_{i} \in \mathbb{R}^{n \times n_{y}}$ and a positive scalar c minimizing $\gamma$ under the LMI constraints (12)

$$
\begin{gathered}
X \geq \alpha_{1} I \\
c-\alpha \alpha_{1} \gamma \leq 0 \\
\sum_{j \in \mathcal{P}(i)}\left(\begin{array}{rr}
\mathbb{S}\left(X \mathcal{A}_{j_{1}}-\bar{K}_{j_{1}} C_{j_{2}}\right)+\alpha X & X \mathcal{E}_{j_{1}} \\
\mathcal{E}_{j_{1}}^{T} X & -c I
\end{array}\right)<0, \forall i \in \mathbb{I}_{p, r}^{+}
\end{gathered}
$$

(the notations $j_{1}$ and $j_{2}$ refer to those of the Lemma 2) then the state estimation error $e(t)=\hat{x}(t)-x(t)$ asymptotically converges to an origin-centered ball of radius $\bar{w} \sqrt{c /\left(\alpha \alpha_{1}\right)}$, for any $u(t)$, $f(t), w(t), x(0)$ and $\hat{x}(0)$. The observer gains are given by (11), $K_{i}=X^{-1} \bar{K}_{i}$ for $i \in \mathcal{I}_{r}$ and

$$
\begin{aligned}
N(\rho, \dot{\rho}) & =\mathcal{A}(\rho, \dot{\rho})-K_{\mu} C(\rho) \\
L(\rho, \dot{\rho}) & =K_{\mu}-N(\rho, \dot{\rho}) H(\rho) \\
G(\rho) & =P(\rho) B(\rho)
\end{aligned}
$$

Proof. The state estimation error between the system (4) and the UIO (5) is $e(t)=z(t)-P(\rho) x(t)$. With (11a) and (11b), its time derivative $\dot{e}(t)$ is

$$
\begin{aligned}
\dot{e}(t)= & N(\rho, \dot{\rho}) e(t)+(-\dot{P}(\rho, \dot{\rho})+N(\rho, \dot{\rho}) P(\rho)+L(\rho, \dot{\rho}) C(\rho) \\
& -P(\rho) A(\rho)) x(t)+(G(\rho)-P(\rho) B(\rho)) u(t) \\
& -P(\rho) F(\rho) f(t)-P(\rho) E(\rho) w(t)
\end{aligned}
$$

Since the Assumption 2 is satisfied, the pseudo inverse of $C(\rho) F(\rho)$ exists and is defined by $(C(\rho) F(\rho))^{+}=$ $\left((C(\rho) F(\rho))^{T} C(\rho) F(\rho)\right)^{-1}(C(\rho) F(\rho))^{T}$. It follows, with (11a) and (11b), that $P(\rho) F(\rho)=0$. From (13c), it also follows that $G(\rho)-P(\rho) B(\rho)=0$. From (11c), (13b) and (13a), it finally follows that $(-\dot{P}(\rho, \dot{\rho})+N(\rho, \dot{\rho}) P(\rho)+L(\rho, \dot{\rho}) C(\rho)-P(\rho) A(\rho))=0$. One should note here that when the polytopic transformation is applied to $\mathcal{A}(\rho, \dot{\rho})$ the resulting weighting functions $\mu_{i}$ for $i \in \mathcal{I}_{r}$ 
(used for $C(\rho),-P(\rho) E(\rho)$ and $\mathcal{A}(\rho, \dot{\rho}))$ depend both on the parameter $\rho(t)$ and on its time derivative $\dot{\rho}(t)$. The state estimation error dynamics is then reduced to

$$
\dot{e}(t)=\left(\mathcal{A}_{\mu}-K_{\mu} C_{\mu}\right) e(t)+\mathcal{E}_{\mu} w(t)
$$

Defining the Lyapunov function by $V(e(t))=e^{T}(t) X e(t)$, its derivative along the trajectory of (15) is given by

$$
\dot{V}(e(t))=e^{T}(t) \mathbb{S}\left(X\left(\mathcal{A}_{\mu}-K_{\mu} C_{\mu}\right)\right) e(t)+2 e^{T}(t) X \mathcal{E}_{\mu} w(t)
$$

Thus, for any real $\alpha$ and with $\bar{K}_{i}=X K_{i}$, it can be written as

$$
\begin{aligned}
\dot{V}(e(t))= & \left(\begin{array}{c}
e(t) \\
w(t)
\end{array}\right)^{T}\left(\begin{array}{cc}
\mathbb{S}\left(X \mathcal{A}_{\mu}-\bar{K}_{\mu} C_{\mu}\right)+\alpha X & X \mathcal{E}_{\mu} \\
\mathcal{E}_{\mu}^{T} X & -c I
\end{array}\right)\left(\begin{array}{c}
e(t) \\
w(t)
\end{array}\right) \\
& -\alpha e^{T}(t) X e(t)+c w^{T}(t) w(t)
\end{aligned}
$$

From Lemma 2 and (17), (12c) implies

$$
\dot{V}(e(\tau))<-\alpha V(e(\tau))+c w^{T}(\tau) w(\tau), \forall \tau \in \mathbb{R}^{+}
$$

Multiplying (18) by $e^{\alpha \tau}$ and integrating from 0 to $t$, it follows

$$
V(e(t)) e^{\alpha t}-V(e(0))<c \int_{0}^{t} w^{T}(\tau) w(\tau) e^{\alpha \tau} d \tau
$$

Under Assumption 3, (19) allows to bound $V(e(t))$ according to

$$
V(e(t))<V(e(0)) e^{-\alpha t}+\frac{c \bar{w}^{2}}{\alpha}
$$

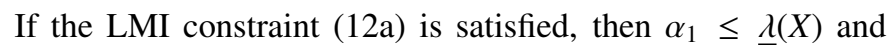
from the definition of $V(e(t))$ it obviously follows that $\bar{V}(e(t)) \geq$ $\underline{\lambda}(X)\|e(t)\|_{2}^{2}$. Consequently, $e(t)$ can be bounded by

$$
\|e(t)\|_{2}^{2}<\frac{V(e(0))}{\alpha_{1}} e^{-\alpha t}+\frac{c \bar{w}^{2}}{\alpha \alpha_{1}}
$$

From the inequality $\sqrt{a+b} \leq \sqrt{a}+\sqrt{b}$, it finally follows that

$$
\|e(t)\|_{2}<\sqrt{\frac{V(e(0))}{\alpha_{1}}} e^{-\alpha t / 2}+\bar{w} \sqrt{\frac{c}{\alpha \alpha_{1}}}
$$

Finally (22) proves that $e(t)$ asymptotically converges to an origin centered ball of radius $\left(\bar{w} \sqrt{c /\left(\alpha \alpha_{1}\right)}\right)$. This radius is minimized with $\gamma$, since (12b) implies $c /\left(\alpha \alpha_{1}\right) \leq \gamma$.

Remark 2. As mentioned in the Remark 2 of Guerra et al. (2015), the matrix inequalities (12) are LMI for fixed $\alpha$ and $\alpha_{1}$, then to avoid any optimization technique when searching for $\alpha$ and $\alpha_{1}$, logarithmically spaced searches can be used, as proposed in Guerra et al. (2015) and in the references therein.

Remark 3. When applying the polytopic transformation, the number of submodels $r$ increases with the number of nonlinearities. If the number of nonlinearities in $\rho$ and $\dot{\rho}$ in (5) is $m$, then the number of submodels is $r=2^{m}$. Nevertheless an adequate choice of the nonlinearities used in the polytopic transformation allows to limit this drawback, as discussed in Nagy et al. (2010) (e.g. in Example 3, even if the system is a third order nonlinear system with $\rho$ simultaneously involved in $A(\rho), F(\rho)$ and $C(\rho)$, the computational burden is reasonable and only 2 gains are to be computed). The problem of the induced conservatism is quite usual in the TS or polytopic formalism and it is partially counterbalanced by the use of the relaxed LMI conditions of the Lemma 2. This lemma, borrowed from Sala and Ariño (2007), is the only necessary and sufficient result of the literature and it allows to obtain a solution even for a large number of submodels and LMI conditions. Unfortunately, the "necessary" property is only obtained for $p \rightarrow \infty$, and in this case the number of LMI conditions also tends to infinity and is untractable. Consequently, the choice of $p$ is a trade-off between conservatism reduction and numerical burden. A practical solution consists in seeking a solution to the LMI problem for $p=2$ and then increasing $p$ if no solution is found.

Remark 4. In Ichalal et al. (2015) a similar ISS result was established for LPV systems affected by measurement noises, requiring not only this measurement noise but also its time derivative to be bounded. These two results can easily be combined to deal with systems encompassing UI w(t) that cannot be perfectly decoupled both on state and output equations that are described by

$$
\begin{aligned}
& \dot{x}(t)=A(\rho) x(t)+B(\rho) u(t)+F(\rho) f(t)+E^{x}(\rho) w(t) \\
& y(t)=C(\rho) x(t)+E^{y}(\rho) w(t)
\end{aligned}
$$

Theorem 1 can be slightly modified to design the UIO. From (5) and (23), and defining the observer gains by (11) and (13), the state estimation error is

$$
\dot{e}(t)=\left(\mathcal{A}_{\mu}-K_{\mu} C_{\mu}\right) e(t)+\mathbb{E}(\rho, \dot{\rho})\left(\begin{array}{l}
w(t) \\
\dot{w}(t)
\end{array}\right)
$$

where $\mathbb{E}(\rho, \dot{\rho})=\left[\mathbb{E}^{1}(\rho, \dot{\rho}) \mathbb{E}^{2}(\rho)\right]$, with $\mathbb{E}^{1}(\rho, \dot{\rho})=K_{\mu} E^{y}(\rho)-$ $P(\rho) E^{x}(\rho)-\dot{H}(\rho, \dot{\rho}) E^{y}(\rho)-H(\rho) \dot{E}^{y}(\rho, \dot{\rho})$ and $\mathbb{E}^{2}(\rho)=$ $-H(\rho) E^{y}(\rho)$. The only difference between (15) and (24) is the substitution of $\mathcal{E}(\rho, \dot{\rho})$ and $w$ by $\mathbb{E}(\rho, \dot{\rho})$ and $\left[w^{T} \dot{w}^{T}\right]^{T}$ respectively. Provided that $w(t)$ satisfies $\|w(t)\|_{2}^{2}+\|\dot{w}(t)\|_{2}^{2}<\bar{w}, \forall t \geq 0$, it suffices to define $\mathcal{E}_{\mu}$ as the polytopic rewriting of $\mathbb{E}(\rho, \dot{\rho})$.

If the disturbance UI $w(t)$ is identically null, the observer (5) can be designed to ensure the state estimation error convergence toward zero. Moreover, the asymptotical UI estimation can be derived. These results are detailed in the following corollaries.

Corollary 1. Provided that the system (4) satisfies the Assumptions 1, 2 and $w(t)=0$, define the parameter dependent matrices $H(\rho), P(\rho)$ and $\mathcal{A}(\rho, \dot{\rho})$ by (11) and apply the polytopic transformation to $C(\rho), \mathcal{A}(\rho, \dot{\rho})$ and $-P(\rho) E(\rho)$ to obtain: $\mathcal{A}(\rho, \dot{\rho})=\mathcal{A}_{\mu}, C(\rho)=C_{\mu}$ and $-P(\rho) E(\rho)=\mathcal{E}_{\mu}$. For a given positive integer $p \geq 2$, if there exists a symmetric positive definite matrix $X \in \mathbb{R}^{n \times n}$ and matrices $\bar{K}_{i} \in \mathbb{R}^{n \times n_{y}}$ satisfying (25)

$$
\sum_{j \in \mathcal{P}(i)} \mathbb{S}\left(X \mathcal{A}_{j_{1}}-\bar{K}_{j_{1}} C_{j_{2}}\right)<0, \forall i \in \mathbb{I}_{p, r}^{+}
$$

then the state estimation error $e(t)=\hat{x}(t)-x(t)$ asymptotically converges towards zero for any $u(t), f(t), x(0)$ and $\hat{x}(0)$. The observer gains are given by (11a), $K_{i}=X^{-1} \bar{K}_{i}$ for $i \in \mathcal{I}_{r}$ and (13). 
Proof. Similarly to the proof of Theorem 1, it follows that if $w(t)=0$, then the state estimation error dynamics reduces to $\dot{e}(t)=N(\rho, \dot{\rho}) e(t)$. Also derived from the previous proof with $w(t)=0, c=0$ and $\alpha=0$, it follows that (25) implies $\dot{V}(e(t))<$ 0 and the asymptotical convergence of $e(t)$ towards zero.

Corollary 2. If $w(t)=0$ and if the UIO (5) is designed according to Corollary 1, an asymptotic estimation of the unknown input is obtained by

$$
\begin{aligned}
\hat{f}(t)= & (C(\rho) F(\rho))^{+}(\dot{y}(t)-(\dot{C}(\rho, \dot{\rho})+C(\rho) A(\rho)) \hat{x}(t) \\
& -C(\rho) B(\rho) u(t))
\end{aligned}
$$

Proof. Let us denote the UI estimation error by $e_{f}(t)=\hat{f}(t)-$ $f(t)$. Differentiating (4b), and since $C(\rho) F(\rho)$ is pseudo invertible, $f(t)$ can be given by

$$
\begin{aligned}
f(t)= & (C(\rho) F(\rho))^{+}(\dot{y}(t)-(\dot{C}(\rho, \dot{\rho})+C(\rho) A(\rho)) x(t) \\
& -C(\rho) B(\rho) u(t))
\end{aligned}
$$

From (26) and (27), the UI estimation error is defined by

$$
e_{f}(t)=-(C(\rho) F(\rho))^{+}(\dot{C}(\rho, \dot{\rho})+C(\rho) A(\rho)) e(t)
$$

The asymptotic convergence of $e(t)$, established in Corollary 1 , implies the asymptotic convergence toward zero of $e_{f}(t)$.

\section{Illustrative examples}

Three examples are provided to illustrate the proposed UIO contributions. In the Example 1 it is shown that even with LTI output equation our UIO design outperforms the ones of Chadli and Karimi (2013) and of Marx et al. (2007) that cannot be applied. Example 2 illustrates the fact that, contrarily to the existing literature, an UIO can be designed for systems with LPV output. Example 3 presents a concrete application to the Lorenz chaotic circuit estimation for secure communications.

Example 1. Let us consider the LPV system (4) with LTI output equation defined by $C=\left(\begin{array}{ll}1 & 1\end{array}\right), E(\rho)=\left(\begin{array}{ll}0 & 0\end{array}\right)^{T}$ and

$$
A(\rho(t))=\left(\begin{array}{cc}
\rho(t) & 6 \\
-1-\rho(t) & -5
\end{array}\right) \quad B(\rho(t))=\left(\begin{array}{c}
\frac{2}{\rho(t)} \\
0
\end{array}\right) \quad F(\rho(t))=\left(\begin{array}{c}
\rho(t) \\
1
\end{array}\right)
$$

with the time varying parameter bounded by: $2 \leq \rho(t) \leq 4$ and $-1 \leq \dot{\rho}(t) \leq 1$.

Classical UIO design. Using the polytopic transformation, $\rho(t)$ can be written as: $\rho(t)=\bar{\rho} h_{1}(\rho(t))+\underline{\rho} h_{2}(\rho(t))$ with $\bar{\rho}=4, \underline{\rho}=2$, $h_{1}(t)=\frac{\rho(t)-\underline{\rho}}{\bar{\rho}-\rho}, h_{2}(t)=\frac{\bar{\rho}-\rho(t)}{\bar{\rho}-\underline{\rho}}$ and where $h_{1}$ and $h_{2}$ satisfy $(1)$. Similarly, $\overline{2} \bar{\rho}(t) \in[0.51] \bar{c}$ can be written as: $2 / \rho(t)=h_{3}(t)+$ $0.5 h_{4}(t)$ with $h_{3}(t)=\frac{2 / \rho(t)-0.5}{0.5}$ and $h_{4}(t)=\frac{1-2 / \rho(t)}{0.5}$, where $h_{3}$ and $h_{4}$ satisfy (1). The system (4) becomes a polytopic one given by (6), with an LTI output equation defined by $C_{1}=C_{2}=C_{3}=$ $C_{4}=\left(\begin{array}{ll}1 & 1\end{array}\right)$ and with the matrix vertices defined by $B_{1}^{T}=B_{3}^{T}=$ (10), $B_{2}^{T}=B_{4}^{T}=(0.50), F_{1}^{T}=F_{2}^{T}=(41), F_{3}^{T}=F_{4}^{T}=(21)$, $E_{1}=E_{2}=E_{3}=E_{4}=\left(\begin{array}{ll}0 & 0\end{array}\right)^{T}, A_{2}=A_{1}$ and $A_{4}=A_{3}$ with

$$
A_{1}=\left(\begin{array}{cc}
4 & 6 \\
-5 & -5
\end{array}\right) \quad A_{3}=\left(\begin{array}{cc}
2 & 6 \\
-3 & -5
\end{array}\right)
$$

The functions $\mu_{i}$ satisfying (1) are defined by: $\mu_{1}=h_{1} h_{3}$, $\mu_{2}=h_{1} h_{4}, \mu_{3}=h_{2} h_{3}$ and $\mu_{4}=h_{2} h_{4}$. The decoupling condition of Chadli and Karimi (2013), i.e. $\operatorname{rank}\left(C\left[\begin{array}{llll}F_{1} & F_{2} & F_{3} & F_{4}\end{array}\right]\right)=$ $\operatorname{rank}\left(\left[\begin{array}{llll}F_{1} & F_{2} & F_{3} & F_{4}\end{array}\right]\right)$ is not satisfied and then the previous UIO designs for LPV systems cannot be applied.

New UIO design. The time varying parameter and its derivative and the system matrices are bounded and thus satisfy the Assumption 1. Assumption 2 is also readily satisfied. Applying the Corollary 1, it follows that

$$
H(\rho(t))=\left(\begin{array}{l}
\frac{-\rho(t)}{1+\rho(t)} \\
\frac{-1}{1+\rho(t)}
\end{array}\right) \quad P(\rho(t))=\left(\begin{array}{ll}
\frac{1}{1+\rho(t)} & \frac{-\rho(t)}{1+\rho(t)} \\
\frac{-1}{1+\rho(t)} & \frac{\rho(t)}{1+\rho(t)}
\end{array}\right)
$$

Then the matrix $\mathcal{A}(\rho(t), \dot{\rho}(t))$ is given by

$$
\mathcal{A}(\rho(t), \dot{\rho}(t))=\left(\begin{array}{ll}
\frac{-\dot{\rho}(t)}{(1+\rho(t))^{2}}+\frac{\rho(t)}{1+\rho(t)}+\rho(t) & \frac{-\dot{\rho}(t)}{(1+\rho(t))^{2}}+\frac{1}{1+\rho(t)}+5 \\
\frac{\dot{\rho}(t)}{(1+\rho(t))^{2}}-\frac{1}{1+\rho(t)}-\rho(t) & \frac{\rho(t)}{(1+\rho(t))^{2}}-\frac{1}{1+\rho(t)}-5
\end{array}\right)
$$

Assumption 4 is readily satisfied. Since $C$ is constant, the polytopic transformation is only applied to $\mathcal{A}(\rho, \dot{\rho})$. It is performed by choosing the following premise variables $\xi_{1}(t)=\frac{-\dot{\rho}(t)}{(1+\rho(t))^{2}}+$ $\frac{\rho(t)}{1+\rho(t)}+\rho(t)$ and $\xi_{2}(t)=\frac{\dot{\rho}(t)}{(1+\rho(t))^{2}}-\frac{1}{1+\rho(t)}$. Exploiting the bounds $\rho(t)$ and $\dot{\rho}(t)$, the following bounds on the premise variables are deduced: $\underline{\xi}_{1} \leq \xi_{1} \leq \bar{\xi}_{1}$ and $\underline{\xi}_{2} \leq \xi_{2} \leq \bar{\xi}_{2}$ with $\underline{\xi}_{1}=23 / 9$, $\bar{\xi}_{1}=221 / 45, \underline{\xi}_{2}=-4 / 9$ and $\bar{\xi}_{2}=-4 / 45$. The weighting functions and the vertex matrices of the polytopic writing of $\mathcal{A}(\rho, \dot{\rho})$ are easily deduced from: $\xi_{i}(t)=\underline{\xi}_{i}\left(\frac{\bar{\xi}_{i}-\xi_{i}(t)}{\bar{\xi}_{i}-\underline{\xi}_{i}}\right)+\bar{\xi}_{i}\left(\frac{\xi_{i}(t)-\underline{\xi}_{i}}{\bar{\xi}_{i}-\underline{\xi}_{i}}\right)$ (for $i=1,2)$ and are given by $\mu_{1}(\rho, \dot{\rho})=\left(\frac{\xi_{1}(t)-\underline{\xi}_{1}}{\bar{\xi}_{1}-\underline{\xi}_{1}}\right)\left(\frac{\bar{\xi}_{2}-\xi_{2}(t)}{\bar{\xi}_{2}-\underline{\xi}_{2}}\right)$, $\mu_{2}(\rho, \dot{\rho})=\left(\frac{\bar{\xi}_{1}-\xi_{1}(t)}{\bar{\xi}_{1}-\underline{\xi}_{1}}\right)\left(\frac{\bar{\xi}_{2}-\xi_{2}(t)}{\bar{\xi}_{2}-\underline{\xi}_{2}}\right), \mu_{3}(\rho, \dot{\rho})=\left(\frac{\xi_{1}(t)-\underline{\xi}_{1}}{\bar{\xi}_{1}-\underline{\xi}_{1}}\right)\left(\frac{\xi_{2}(t)-\underline{\xi}_{2}}{\bar{\xi}_{2}-\underline{\xi}_{2}}\right)$, $\mu_{4}(\rho, \dot{\rho})=\left(\frac{\bar{\xi}_{1}-\xi_{1}(t)}{\bar{\xi}_{1}-\underline{\xi}_{1}}\right)\left(\frac{\xi_{2}(t)-\underline{\xi}_{2}}{\bar{\xi}_{2}-\underline{\xi}_{2}}\right)$ and

$$
\begin{aligned}
& \mathcal{A}_{1}=\left(\begin{array}{cc}
\bar{\xi}_{1} & 5-\underline{\xi}_{2} \\
-\bar{\xi}_{1} & \underline{\xi}_{2}-5
\end{array}\right) \mathcal{A}_{2}=\left(\begin{array}{cc}
\underline{\xi}_{1} & 5-\underline{\xi}_{2} \\
-\underline{\xi}_{1} & \underline{\xi}_{2}-5
\end{array}\right) \\
& \mathcal{A}_{3}=\left(\begin{array}{cc}
\bar{\xi}_{1} & 5-\bar{\xi}_{2} \\
-\bar{\xi}_{1} & \bar{\xi}_{2}-5
\end{array}\right) \mathcal{A}_{4}=\left(\begin{array}{cc}
\underline{\xi}_{1} & 5-\bar{\xi}_{2} \\
-\underline{\xi}_{1} & \bar{\xi}_{2}-5
\end{array}\right)
\end{aligned}
$$

The solution of the LMI problem is

$$
K_{1}=\left(\begin{array}{c}
5.43 \\
-4.93
\end{array}\right) K_{2}=\left(\begin{array}{c}
4.25 \\
-3.75
\end{array}\right) K_{3}=\left(\begin{array}{c}
5.25 \\
-4.75
\end{array}\right) K_{4}=\left(\begin{array}{c}
4.07 \\
-3.57
\end{array}\right)
$$

From the definitions (13), (11c), (1) and the gains (32), the time varying matrices $N(\rho, \dot{\rho}), L(\rho, \dot{\rho})$ and $G(\rho)$ are deduced. The control input $u(t)$, the parameter $\rho(t)$, the output $y(t)$ and the comparison of the original and estimated states and UI are displayed on the Figure 1. One can see that the state and UI estimations are very satisfactory.

Example 2. Now, the UIO design for LPV systems with LPV output equation is illustrated. The considered system is (4) with

$$
\begin{gathered}
A(\rho)=\left(\begin{array}{cc}
-\rho(t) & 0.2 \\
1+\rho(t) & -1
\end{array}\right) \quad B(\rho)=\left(\begin{array}{c}
\frac{2}{\rho(t)} \\
0
\end{array}\right) \quad F(\rho)=\left(\begin{array}{c}
\rho(t) \\
1
\end{array}\right) \\
C(\rho)=\left(\begin{array}{ll}
0 & 1+\rho(t)
\end{array}\right)
\end{gathered}
$$



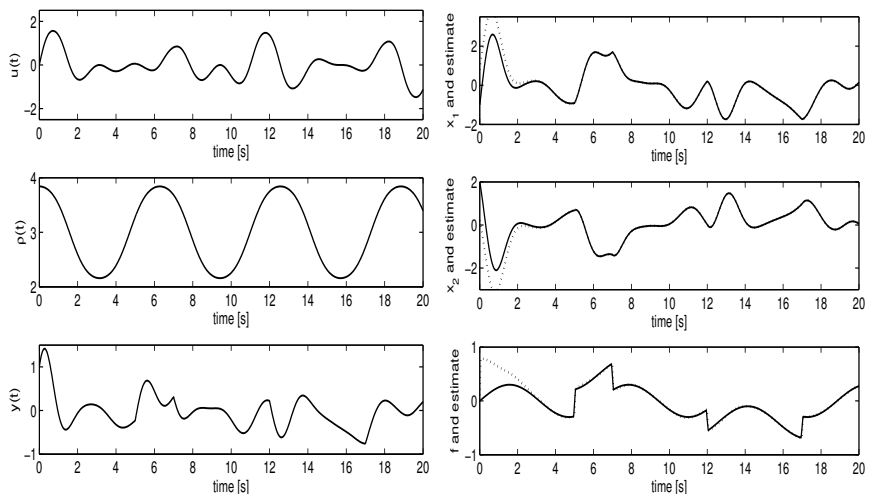

Figure 1: Control input (top left), parameter (middle left) and output (bottom left), original (line) and estimated (dashed) state variables (top and middle right) and UI (bottom right)

The parameter and its derivative are bounded by $2 \leq \rho(t) \leq 4$ and $-1 \leq \dot{\rho}(t) \leq 1$ and satisfy the Assumption 1. Applying Corollary 1 it follows

$$
H(\rho)=\left(\begin{array}{c}
\frac{-\rho(t)}{1+\rho(t)} \\
\frac{-1}{1+\rho(t)}
\end{array}\right) \quad P(\rho)=\left(\begin{array}{cc}
1 & -\rho(t) \\
0 & 0
\end{array}\right) \quad G(\rho)=\left(\begin{array}{c}
\frac{2}{\rho(t)} \\
0
\end{array}\right)
$$

The matrices under a polytopic form are $C(\rho(t))$ in (4) and

$$
\mathcal{A}(\rho(t), \dot{\rho}(t))=\left(\begin{array}{cc}
-2 \rho(t)-\rho^{2}(t) & 0.2+\rho(t)-\dot{\rho}(t) \\
0 & 0
\end{array}\right)
$$

The premise variables are $\xi_{1}(t)=2 \rho(t)+\rho^{2}(t), \xi_{2}(t)=\rho(t)-\dot{\rho}(t)$ and $\xi_{3}(t)=\rho(t)$. Similarly to the previous example, their lower and upper bounds are: $\xi_{1}=8, \bar{\xi}_{1}=24, \xi_{2}=1, \bar{\xi}_{1}=5$, $\underline{\xi}_{3}=2$ and $\bar{\xi}_{3}=4$ and the $r=8$ weighting functions satisfying (1) are defined by $\mu_{1}(\rho, \dot{\rho})=\left(\frac{\xi_{1}(t)-\underline{\xi}_{1}}{\bar{\xi}_{1}-\underline{\xi}_{1}}\right)\left(\frac{\bar{\xi}_{2}-\xi_{2}(t)}{\bar{\xi}_{2}-\underline{\xi}_{2}}\right)\left(\frac{\bar{\xi}_{3}-\xi_{3}(t)}{\bar{\xi}_{3}-\underline{\xi}_{3}}\right), \ldots$, $\mu_{8}(\rho, \dot{\rho})=\left(\frac{\bar{\xi}_{1}-\xi_{1}(t)}{\bar{\xi}_{1}-\underline{\xi}_{1}}\right)\left(\frac{\xi_{2}(t)-\underline{\xi}_{2}}{\bar{\xi}_{2}-\underline{\xi}_{2}}\right)\left(\frac{\xi_{3}(t)-\underline{\xi}_{3}}{\bar{\xi}_{3}-\underline{\xi}_{3}}\right)$. The matrices $\mathcal{A}_{i}$ and $C_{i}$ are

$$
\begin{array}{ll}
\mathcal{A}_{1}=\left(\begin{array}{cc}
-24 & 1.2 \\
0 & 0
\end{array}\right) & \mathcal{A}_{2}=\left(\begin{array}{cc}
-8 & 1.2 \\
0 & 0
\end{array}\right) \\
\mathcal{A}_{3}=\left(\begin{array}{cc}
-24 & 5.2 \\
0 & 0
\end{array}\right) & \mathcal{A}_{4}=\left(\begin{array}{cc}
-8 & 5.2 \\
0 & 0
\end{array}\right)
\end{array}
$$

$C_{1}=\left(\begin{array}{ll}0 & 3\end{array}\right), C_{5}=\left(\begin{array}{ll}0 & 5\end{array}\right), \mathcal{A}_{5}=\mathcal{A}_{1}, \mathcal{A}_{6}=\mathcal{A}_{2}, \mathcal{A}_{7}=\mathcal{A}_{3}$, $\mathcal{A}_{8}=\mathcal{A}_{4}, C_{2}=C_{1}, C_{3}=C_{1}, C_{4}=C_{1}, C_{6}=C_{5}, C_{7}=C_{5}$ and $C_{8}=C_{5}$. The obtained $K_{i}$ are

$$
\begin{aligned}
& K_{1}=\left(\begin{array}{l}
0.9794 \\
0.0772
\end{array}\right) K_{2}=\left(\begin{array}{l}
0.7014 \\
0.0770
\end{array}\right) K_{3}=\left(\begin{array}{l}
1.9664 \\
0.0772
\end{array}\right) K_{4}=\left(\begin{array}{l}
1.6884 \\
0.0770
\end{array}\right) \\
& K_{5}=\left(\begin{array}{l}
0.5198 \\
0.0489
\end{array}\right) K_{6}=\left(\begin{array}{l}
0.2654 \\
0.0487
\end{array}\right) K_{7}=\left(\begin{array}{l}
1.4230 \\
0.0489
\end{array}\right) K_{8}=\left(\begin{array}{l}
1.1686 \\
0.0487
\end{array}\right)
\end{aligned}
$$

The control input $u(t)$, the time varying parameter $\rho(t)$, the output signal $y(t)$ and the comparison of the original and estimated state variables and UI are displayed on the Figure 2. It can be seen that even if the output equation depends on the parameter, the state and unknown estimations are correct.
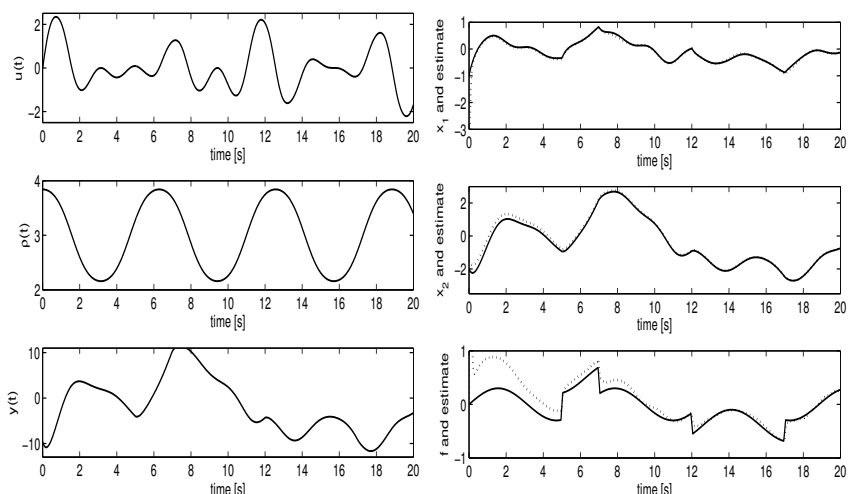

Figure 2: Control input (top left), parameter (middle left) and output (bottom left), original (line) and estimated (dashed) state variables (top and middle right) and UI (bottom right)

Example 3. In this third example, a system widely used for secure communication is considered (Cuomo et al., 1993). This system is the Lorenz model defined by

$$
\left\{\begin{array}{l}
\dot{x}_{1}(t)=a\left(x_{2}(t)-x_{1}(t)\right) \\
\dot{x}_{2}(t)=b x_{1}(t)-x_{2}(t)-x_{1}(t) x_{3}(t)+\left(x_{1}(t)+200\right) f(t) \\
\dot{x}_{3}(t)=x_{1}(t) x_{2}(t)-c x_{3}(t) \\
y_{1}(t)=x_{1}(t) \\
y_{2}(t)=\left(x_{1}(t)+40\right) x_{2}(t)+x_{3}(t)
\end{array}\right.
$$

where $a=16, b=45.6$ and $c=-4$. The UI is the information to encrypt and securely transmit. Introducing the UI in a nonlinear way and considering nonlinear output to transmit may enhance the encryption of the informations. By considering the measurable varying parameter $\rho(t)=y_{1}(t)$, the LPV system (4) is defined by $B(\rho)=0, E(\rho)=0$ and

$$
\begin{gathered}
A(\rho)=\left(\begin{array}{ccc}
-a & a & 0 \\
b & -1 & -\rho(t) \\
0 & \rho(t) & -c
\end{array}\right) \quad F(\rho)=\left(\begin{array}{c}
0 \\
\rho(t)+200 \\
0
\end{array}\right) \\
C(\rho)=\left(\begin{array}{ccc}
1 & 0 & 0 \\
0 & \rho(t)+40 & 1
\end{array}\right)
\end{gathered}
$$

Assumption 2 is satisfied, and using the proposed approach, one obtains the matrices

$$
H(\rho)=\left(\begin{array}{cc}
0 & 0 \\
0 & -\frac{1}{\rho(t)+40} \\
0 & 0
\end{array}\right) \quad P(\rho)=\left(\begin{array}{lll}
1 & 0 & 0 \\
0 & 0 & 0 \\
0 & 0 & 1
\end{array}\right)
$$

Since $P(\rho)$ is constant, the matrix $\mathcal{A}(\rho, \dot{\rho})$ does not depend on the time derivative of the parameter. In this case, the Assumption 1 is relaxed and the knowledge of $\rho(t)=y_{1}(t)$ is sufficient to satisfy the Assumption 1. The matrix $\mathcal{A}(\rho)$ is then given by

$$
\mathcal{A}(\rho)=\left(\begin{array}{ccc}
-16 & 16 & 0 \\
0 & 0 & 0 \\
0 & \rho(t) & 4
\end{array}\right)
$$

The matrix $N(\rho)$ is then given by $N(\rho)=\mathcal{A}(\rho)-K(\rho) C(\rho)$. Applying the polytopic transformation and using Corollary 1 , 

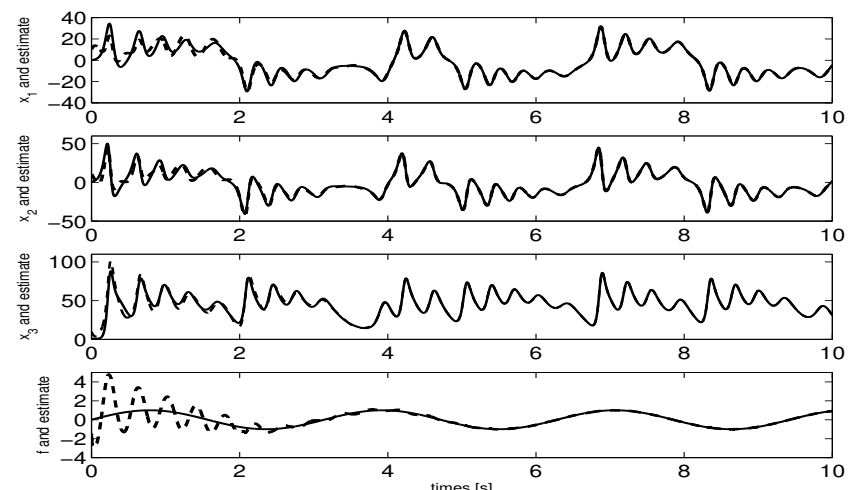

Figure 3: Original (line) and estimated (dashed) state variables (top three figures) and original and estimated UI (bottom)

the obtained gains are

$$
K_{1}=\left(\begin{array}{cc}
-13.80 & -0.001 \\
16.17 & -0.016 \\
0.417 & 0.472
\end{array}\right) \quad K_{2}=\left(\begin{array}{cc}
-13.79 & -0.0024 \\
16.27 & -0.016 \\
0.418 & 0.472
\end{array}\right)
$$

Applying Corollary 2, the UI can be estimated, i.e. the encrypted message can be recovered. The state and the unknown input estimation results are depicted in the Figure 3.

\section{Conclusion}

This paper investigated the problem of unknown input observer design for LPV systems and brought two main contributions. The first one is to relax the known rank conditions needed to UIO design for LPV systems to decouple the estimation and the UI. The second one is that, contrarily to the existing literature, the output equation of the LPV system is not restricted to be linear time invariant. The proposed UIO designs rely on LMI optimization in order to compute the observer gains. In the disturbance free case, state estimation error asymptotically converges to zero and fault estimation is proposed. If disturbances affect the system, the state estimation error is ensured to converge in a bounded ball. Finally, examples are provided in order to illustrate the two main proposed improvements.

\section{References}

Aldeen, M., Sharma, R., 2008. Estimation of states, faults and unknown disturbances in non-linear systems. International Journal of Control 81, 119-1201.

Chadli, M., Karimi, H., 2013. Robust observer design for unknown inputs Takagi-Sugeno models. IEEE Transactions on Fuzzy Systems 21, 158-164.

Cuomo, K., Oppenheim, A., Strogatz, S., 1993. Synchronization of Lorenzbased chaotic circuits with applications to communications. IEEE Transactions on Circuits and Systems II: Analog and Digital Signal Processing 40, 626-633.

Darouach, M., Zasadzinski, M., Xu, S., 1994. Full-order observers for linear systems with unknown inputs. IEEE Transactions on Automatic Control 39, 606-609.

Feng, G., 2006. A survey on analysis and design of model-based fuzzy control systems. IEEE Transactions on Fuzzy Systems 14, 676-697.

Fliess, M., Join, C., Sira-Ramirez, H., 2008. Non-linear estimation is easy. International Journal of Modelling, Identification and Control 4, 12-27.
Gao, Z., Ho, D., 2004. Proportional multiple-integral observer design for descriptor systems with measurement output disturbances. IEE proceeding Control Theory and Application 151, 279-288.

Guerra, T.M., Estrada-Manzo, V., Lendek, Z., 2015. Observer design for Takagi-Sugeno descriptor models: An LMI approach. Automatica 52, 154 159.

Hassanabadi, A., Shafiee, M., Puig, V., 2016. UIO design for singular delayed LPV systems with application to actuator fault detection and isolation. International Journal of Systems Science 47, 107-121.

Heemels, W., Daafouz, J., Millérioux, G., 2010. Observer-based control of discrete-time LPV systems with uncertain parameters. IEEE Transactions on Automatic Control 55, 2130 -2135.

Hou, M., Muller, P., 1994. Disturbance decoupled observer design: a unified viewpoint. IEEE Transactions on Automatic Control 39, 1338-1341.

Ichalal, D., Mammar, M., 2015. On unknown input observers for LPV systems. IEEE Transactions on Industrial Electronics 62, 5870-5880.

Ichalal, D., Marx, B., Ragot, J., Maquin, D., 2009. Simultaneous state and unknown inputs estimation with PI and PMI observers for Takagi-Sugeno model with unmeasurable premise variables, in: 17th Mediterranean Conference on Control and Automation, MED '09, pp. 353-358.

Ichalal, D., Marx, B., Ragot, J., Maquin, D., 2015. Unknown input observer for LPV systems with parameter varying output equation, in: 9th IFAC Symposium on Fault Detection, Supervision and Safety for Technical Processes, SAFEPROCESS 2015, pp. 1030-1035.

Koenig, D., 2006. Observer design for unknown input nonlinear descriptor systems via convex optimization. IEEE Transactions on Automatic Control 51, 047- 1052 .

Lendek, Z., Guerra, T., Babuska, R., De Schutter, B., 2010. Stability analysis and nonlinear observer design using Takagi-Sugeno fuzzy models. Springer.

Levant, A., 2003. Higher-order sliding modes, differentiation and outputfeedback control. International Journal of Control 76, 924-941.

Marx, B., Koenig, D., Ragot, J., 2007. Design of observers for Takagi-Sugeno descriptor systems with unknown inputs and application to fault diagnosis. IET Control Theory and Application 1, 1487-1495.

Nagy, A., Mourot, G., Marx, B., Schutz, G., Ragot, J., 2010. Systematic multimodeling methodology applied to an activated sludge reactor model. Industrial \& Engineering Chemistry Research 46, 2790-2799.

Sala, A., 2009. On the conservativeness of fuzzy and fuzzy-polynomial control of nonlinear systems. Annual Reviews in Control 33, 48-58.

Sala, A., Ariño, C., 2007. Asymptotically necessary and sufficient conditions for stability and performance in fuzzy control: Applications of Polya's theorem. Fuzzy Sets and Systems 158, 2671-2686.

Sename, O., Gaspar, P., Bokor, J., 2013. Robust Control and Linear Parameter Varying approaches: Application to Vehicle Dynamics. Springer.

Sontag, E., Wang, Y., 1995. On characterizations of the input-to-state stability property. Systems \& Control Letters 24, 351-359.

Tan, C., Crusca, F., Aldeen, M., 2008. Extended results on robust state estimation and fault detection. Automatica 44, 2027-2033.

Tanaka, K., Wang, H., 2001. Fuzzy Control Systems Design and Analysis: A Linear Matrix Inequality Approach. John Wiley and Sons.

Tuan, H., Apkarian, P., Narikiyo, T., Yamamoto, Y., 2001. Parameterized linear matrix inequality techniques in fuzzy control system design. IEEE Transactions on Fuzzy Systems 9, 324-332.

Wojciechowski, B., 1978. Analysis and synthesis of proportional-integral observers for single-input-single-output time-invariant continuous systems. Ph.D. thesis. Gliwice, Poland.

Yacine, Z., Ichalal, D., Ait-Oufroukh, N., Mammar, S., Djennoune, S., 2015. Takagi-Sugeno observers: Experimental application for vehicle lateral dynamics estimation. IEEE Transactions on Control Systems Technology 23, 754-761. 